\title{
Natural history of coexistent mitral regurgitation after aortic valve replacement
}

\author{
Sarah A. Schubert, MD, Leora T. Yarboro, MD, Sushma Madala, MD, Karnika Ayunipudi, MD, \\ Irving L. Kron, MD, John A. Kern, MD, Gorav Ailawadi, MD, George J. Stukenborg, PhD, and \\ Ravi K. Ghanta, MD
}

\begin{abstract}
Objectives: The long-term evolution of coexistent mitral regurgitation (MR) after aortic valve replacement (AVR) for aortic stenosis remains poorly defined. Prior studies have demonstrated that acute improvement in MR after AVR is modest, and more aggressive approaches have been advocated. This study examines the evolution of MR after AVR and identifies prognostic indicators for MR improvement.
\end{abstract}

Methods: We retrospectively evaluated demographic and echocardiographic data of 423 patients who underwent primary isolated AVR for aortic stenosis with coexistent mild $(n=314)$ or moderate $(n=109)$ MR at our institution, from 2004 to 2013. For each patient, preoperative and postoperative MR was extracted from 903 echocardiograms and graded on a 0 to $4+$ scale. Hierarchic linear models were used to estimate postoperative residual MR over a 5-year followup period. Patients were then stratified by improvement in MR, and preoperative risk factors and survival were compared between groups. Cox proportional hazards regression was used to assess the association between survival and preoperative and postoperative MR.

Results: The overall acute reduction in MR was -0.23 degrees per patient. Patients with moderate MR had a -0.53 degree reduction in MR, whereas patients with mild MR had only a -0.13 degree reduction in MR $(P<.001)$. Residual MR, however, worsened over time and regressed back to baseline, particularly in patients with preoperative moderate MR. At last follow-up, 70 (17\%) patients returned to $2+$ or worse MR. Residual MR at last echocardiographic follow-up was not affected by left ventricular ejection fraction, severity of preoperative aortic valve gradient (AVG), magnitude of reduction of AVG, or other comorbidities. Degree of preoperative MR did not affect midterm survival. Patients whose MR improved after AVR demonstrated a trend toward improved survival $(75 \%$ vs $65 \%$ 5-year survival; $P=.06$ ), compared with those without MR whose survival remained unchanged or worsened.

Conclusions: Coexistent MR modestly improves after AVR, but eventually regresses back to baseline or worsens over time in many patients. Preoperative $\mathrm{AVG}$, reduction of AVG, heart failure, or atrial fibrillation was not predictive of residual MR. Moderate preoperative MR did not adversely affect 5-year survival. Patients with improvement in MR, however, demonstrated a trend toward improved survival at 5 years. More aggressive approaches for coexistent moderate MR should be considered in patients who need AVR for aortic stenosis. (J Thorac Cardiovasc Surg 2016;151:1032-42)

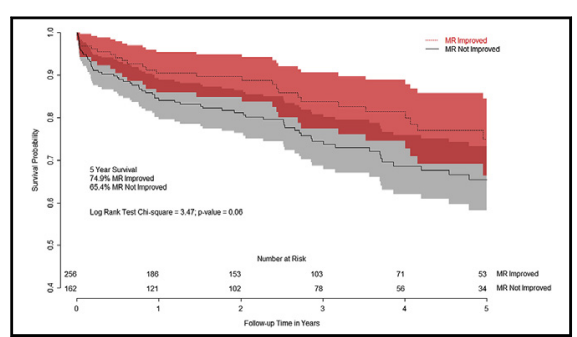

Survival analysis of patients with unchanged or improved MR after AVR, compared with those whose MR progressed. Survival at 5 years postoperatively was significantly better for patients with unchanged or improved MR, compared with those patients whose MR worsened. Long-term survival was significantly better for patients with improved MR, compared with those with worse MR. Shaded areas bounding the estimated functions for each group show 95\% confidence intervals.

\section{Central Message}

Patients with less MR after AVR had better long-term survival. More aggressive treatment of MR should be considered at the time of AVR.

\section{Perspective}

The long-term evolution of coexistent MR after AVR for aortic stenosis is poorly defined. Severe MR mandates concomitant mitral valve surgery; however, optimal management of intermediate degrees of MR at the time of AVR remains unclear. We sought to examine the evolution of residual MR after AVR for aortic stenosis, identify prognostic indicators for MR improvement, and evaluate the effect of residual MR on survival.

See Editorial Commentary page 1043.
From the Department of Thoracic and Cardiovascular Surgery, University of Virginia, Charlottesville, Va.

Read at the 41st Annual Meeting of The Western Thoracic Surgical Association, Whistler, British Columbia, Canada, June 24-27, 2015.

Received for publication June 29, 2015; revisions received Nov 23, 2015; accepted for publication Dec 3, 2015; available ahead of print Feb 11, 2016.
Address for reprints: Ravi K. Ghanta, MD, Department of Surgery, Division of Thoracic and Cardiovascular Surgery, University of Virginia School of Medicine, PO Box 800679, Charlottesville, VA 22908 (E-mail: rghanta@virginia.edu). $0022-5223 / \$ 36.00$

Copyright () 2016 by The American Association for Thoracic Surgery http://dx.doi.org/10.1016/j.jtcvs.2015.12.006 


\section{Abbreviations and Acronyms \\ $\mathrm{AVG}=$ aortic valve gradient \\ $\mathrm{AVR}=$ aortic valve replacement \\ $\mathrm{MR}=$ mitral regurgitation}

Scanning this QR code will take you to supplemental figure for this article.

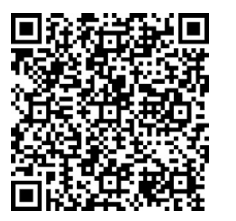

Mitral regurgitation (MR) is frequently observed in patients who have aortic stenosis who present for aortic valve replacement (AVR). ${ }^{1}$ Severe MR mandates concomitant mitral valve surgery; however, optimal management of intermediate degrees of MR at the time of AVR remains unclear. ${ }^{2}$ A widely held assumption is that MR should decrease after AVR, as relief of the AVG should decrease the left ventricular pressure and thereby reduce the transmitral gradient and the hemodynamic provocation for MR.

However, wide variability and inconsistency is found in AVR effects on MR, especially in patients who have lesser degrees of MR. The etiology of MR in patients with aortic stenosis is frequently functional, secondary to ventricular and annular dilatation. Functional MR has been reported in as high as $75 \%$ of patients who undergo AVR. ${ }^{3}$ Kaczorowski and colleagues ${ }^{4}$ recently found that the acute quantitative change in MR is very modest and have suggested that more aggressive concomitant mitral valve surgery be considered. The mortality with double valve surgery may be significantly higher than that with isolated AVR; thus, the addition of concomitant mitral valve surgery must be considered carefully.

Moreover, the effect of residual MR on survival and development of heart failure is unclear. Ruel and colleagues $^{5}$ found that preoperative MR did not adversely affect survival, unless other signs of heart failure, such as left atrial enlargement, were present. No prior study has evaluated the effect of residual MR on survival. In this study, we had the following aims: to examine the evolution of residual MR after AVR for aortic stenosis, identify prognostic indicators for MR improvement, and evaluate the effect of residual MR on survival. We compare the outcomes of patients who have preoperative moderate MR with those who have mild MR.

\section{METHODS \\ Patients}

We queried our Society of Thoracic Surgeons-compliant database at the University of Virginia to identify patients who underwent primary isolated AVR from 2004 to 2013 who had coexistent MR as shown on a preoperative echocardiogram. We limited our study to patients who had primary isolated AVR, with concomitant mild or moderate MR, and a postoperative echocardiogram at our institution (Figure 1). Using these selection criteria, we identified 423 patients and further stratified them by degree of preoperative MR: mild $(\mathrm{n}=314)$ or moderate $(\mathrm{n}=109)$ MR. This study was approved the University of Virginia Institutional Review Board, with a waiver of informed consent.

\section{Grading of Mitral Regurgitation}

The degree of MR was extracted from preoperative, intraoperative, and postoperative echocardiogram reports. All patients underwent preoperative transthoracic echocardiography and intraoperative transesophageal echocardiography. Postoperative echocardiograms were obtained at all available time points for each patient. Follow-up echocardiograms were obtained on the patients at variable intervals and at the discretion of the patients' individual cardiologists.

Patients underwent 903 follow-up echocardiograms at the University of Virginia, which were included in our statistical analysis. All echocardiograms were read in a single laboratory, which utilizes effective regurgitant orifice area to determine MR severity. A summary of the frequency and duration of follow-up for echocardiograms obtained for patients during the postoperative period is presented in Figure E1. Grading of MR was as follows: 0 for no regurgitation, 0.5 for trace, $1+$ for mild, $2+$ for moderate, $3+$ for moderate-severe, and $4+$ for severe, as defined by the American Society of Echocardiography. ${ }^{6}$

A change in the degree of MR was calculated by subtracting the grade of preoperative MR from the grade of postoperative MR. An acute change in MR was determined by the difference seen on preoperative echocardiogram versus intraoperative transesophageal echocardiogram. In addition to degree of MR, the left ventricular ejection fraction and peak AVG data were extracted from all echocardiographic data and utilized for risk assessment.

\section{Outcomes}

We sought to address the following questions:

1. How much does MR improve acutely after AVR for aortic stenosis?

2. How does residual MR evolve over time after AVR?

3. Do any hemodynamic factors predict residual MR?

4. Does residual MR affect patient survival?

\section{Statistical Analysis}

To evaluate residual MR over time, we constructed a hierarchic linear model (repeated-measures mixed model; SAS Proc Mixed, version 9.4, SAS Institute, Inc, Cary, NC), utilizing all available postoperative echocardiographic data. ${ }^{7}$ Specifically, a multilevel random coefficient model, with both separate intercepts and slopes for each patient, was used to estimate the postoperative residual MR score over the available follow-up period. To model MR over time, based on preoperative MR grade, we included a patient-level covariate based on its severity (mild or moderate). In addition, we included preoperative AVG and change in AVG (AVG at time of echocardiogram minus preoperative AVG) as covariate predictors to evaluate the relationship of AVG to MR

The model yields fixed-effect estimates of the overall mean residual MR at selected time points over the follow-up period, along with estimates for the difference in mean score by patient group, the change in score over time, and the difference in change in score over time by patient group. The potential for nonlinearity in the functional relationship was assessed 


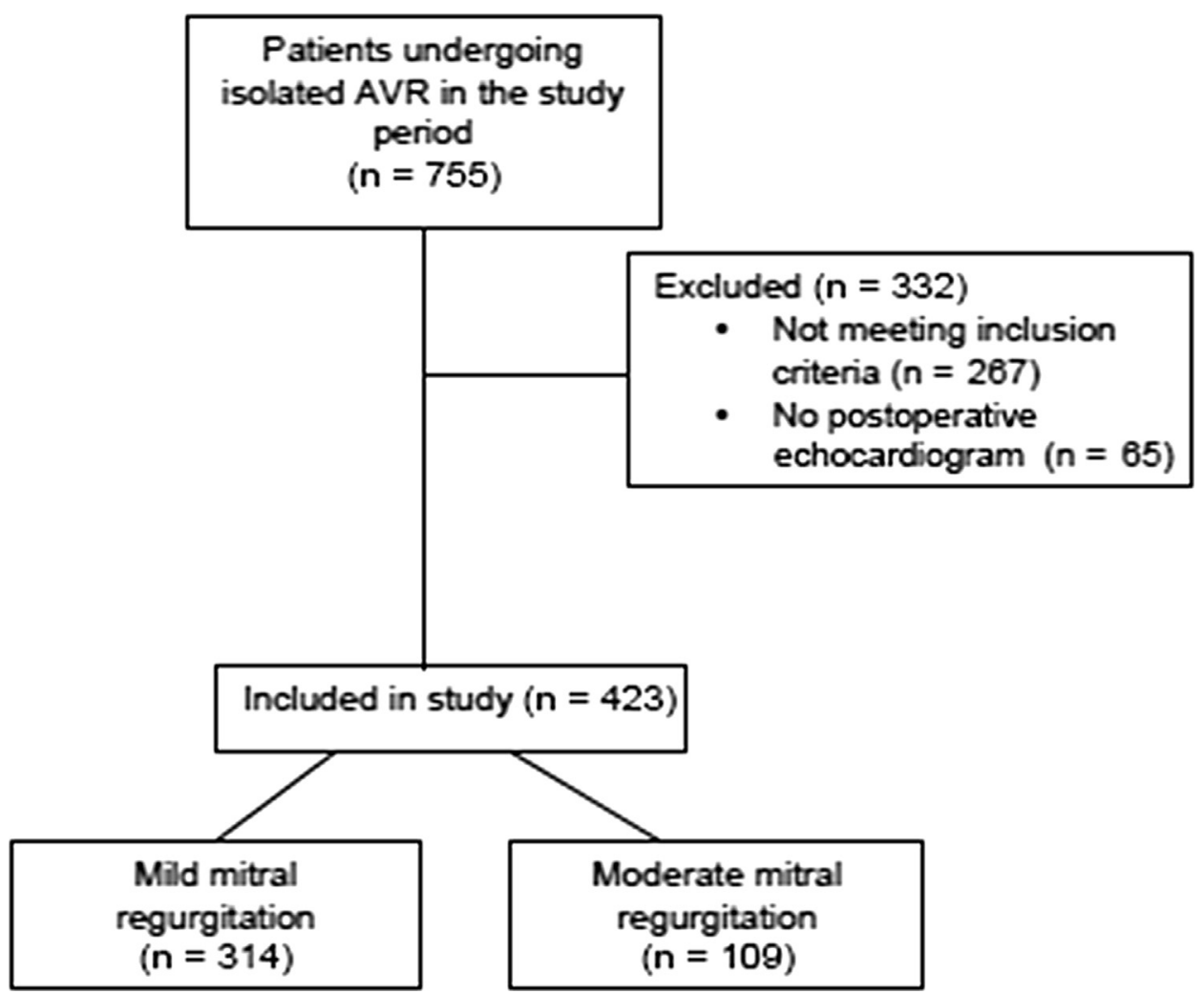

FIGURE 1. Inclusion and exclusion criteria for patients undergoing isolated AVR during the study period. Inclusion criteria: aortic valve replacement, aortic stenosis, mild or greater mitral regurgitation. Exclusion criteria: reoperative procedure, other concomitant operation (ie, coronary artery bypass grafting, aorta, additional valve procedure). $A V R$, Aortic valve replacement.

by including a quadratic term for the effect of follow-up duration in the model. This approach accounts for variation among patients in follow-up number of echocardiograms available and duration. The statistical significance of the difference in the functional relationships over the follow-up period was assessed using the $\mathrm{F}$ test statistic $(P<.05)$.

Patients were further stratified into 2 groups based on whether they demonstrated improvement or no improvement and/or progression of their MR at the last follow-up echocardiogram after AVR. The distributions and frequencies of preoperative risk-factor values between groups were compared, to determine if any significant differences were associated with postoperative improvement or worsening of MR. The statistical significance of differences in continuous variables was assessed using the $t$-test statistic $(P<.05)$, and differences in the proportional distribution of categoric values was assessed using $\chi^{2}$ analysis $(P<.05)$. Cox proportional hazard regression analysis was conducted to assess the statistical significance of differences in the relative hazard of death between patients with preoperative mild versus moderate MR, and between patients with postoperative improved or stable MR versus worse MR. The statistical significance of differences in survival functions calculated for compared groups was assessed using log-rank $\chi^{2}$ analysis $(P<.05)$.

\section{RESULTS}

\section{Patients}

Patient demographic, preoperative, and intraoperative echocardiographic and hemodynamic data are shown in Table 1 . The vast majority of patients had functional MR $(96 \%)$, with very few patients having rheumatic $(3 \%)$ or leaflet prolapse $(1 \%)$ mitral valve pathologies. Patients with preoperative moderate MR were more likely to be women $(53 \%$ vs $36 \% ; P=.02)$ and to have atrial fibrillation $(40 \%$ vs $29 \% ; P=.03)$, congestive heart failure $(70 \%$ vs $50 \% ; P=.01)$, and increased pulmonary artery systolic pressure $(51 \pm 18 \mathrm{~mm} \mathrm{Hg}$ vs $40 \pm 13 \mathrm{~mm} \mathrm{Hg} ; P=.003)$.

\section{Acute Change in MR After AVR}

The overall acute reduction in MR was -0.23 degrees per patient. Reduction for patients with moderate MR was -0.53 degrees. Patients who had mild MR had a reduction in MR of only -0.13 degrees $(P<.001)$.

\section{Evolution of Residual MR Over Time}

The model-estimated trends in postoperative residual MR are plotted in Figure 2, with 95\% confidence intervals for model estimates represented for preoperative mild and moderate MR groups. Initially, MR improved in patients who had moderate or greater MR. By the second postoperative year, however, mean residual MR in these patients began to worsen. Forty-eight $(11.3 \%)$ patients experienced worsening of MR beyond their preoperative baseline in the years after AVR. Patients with mild MR remained stable throughout 5 years of follow-up. 
TABLE 1. Patient demographic and preoperative echocardiographic and hemodynamic data

\begin{tabular}{|c|c|c|c|c|}
\hline Variable & $\begin{array}{c}\text { Overall } \\
\text { MR } \\
(n=423)\end{array}$ & $\begin{array}{c}\text { Preoperative } \\
\text { mild MR } \\
(n=314)\end{array}$ & $\begin{array}{c}\text { Preoperative } \\
\text { moderate } \\
\text { MR } \\
(n=109) \\
\end{array}$ & $P$ value \\
\hline Age (y) & $73 \pm 13$ & $72 \pm 13$ & $75 \pm 14$ & .2 \\
\hline Gender, female & $172(41)$ & $116(36)$ & $56(53)$ & .02 \\
\hline Atrial fibrillation & $133(31)$ & $91(29)$ & $42(40)$ & .03 \\
\hline CHF & $233(55)$ & $160(50)$ & $73(70)$ & .01 \\
\hline Chronic lung disease & $145(34)$ & $113(35)$ & $32(30)$ & .43 \\
\hline PVD & $86(20)$ & $63(20)$ & $23(22)$ & .63 \\
\hline Hypertension & $346(82)$ & $263(82)$ & $83(79)$ & .44 \\
\hline Diabetes & $121(29)$ & $97(30)$ & $24(23)$ & .14 \\
\hline MR etiology & & & & .38 \\
\hline Functional & 407 (96) & $310(97)$ & $98(93)$ & \\
\hline Rheumatic & $12(3)$ & $7(2)$ & $5(5)$ & \\
\hline Leaflet prolapse & $4(1)$ & $2(1)$ & $2(2)$ & \\
\hline \multicolumn{5}{|l|}{ Preoperative } \\
\hline LV EF (\%) & $52.5 \pm 13.4$ & $53.5 \pm 12.6$ & $49.0 \pm 14.0$ & .07 \\
\hline LV EDD (cm) & $4.8 \pm 0.9$ & $4.7 \pm 0.9$ & $5.1 \pm 0.9$ & .48 \\
\hline $\mathrm{AVG}(\mathrm{mm} \mathrm{Hg})$ & $47.9 \pm 15$ & $48.7 \pm 14.8$ & $45.6 \pm 15.7$ & .42 \\
\hline PASP $(\mathrm{mm} \mathrm{Hg})$ & $43.3 \pm 15.3$ & $40.1 \pm 13.0$ & $51.1 \pm 17.9$ & .003 \\
\hline
\end{tabular}

Values are $\mathrm{n}(\%)$, or for continuous variables, mean \pm standard deviation, unless otherwise indicated. Bold indicates statistically significant $P$ values $(P<.05) . M R$, Mitral regurgitation; $C H F$, congestive heart failure; $P V D$, peripheral vascular disease; $L V$, left ventricular; $E F$, ejection fraction; $E D D$, end-diastolic diameter; $A V G$, aortic valve gradient; $P A S P$, pulmonary artery systolic pressure.

\section{Risk Factors for Residual MR}

Table 2 compares preoperative and postoperative risk factors in patients who have improved versus nonimproved MR at last echocardiographic follow-up (median 216 days; 21 days at 25 th percentile; 754 days at 75 th percentile). After AVR, at last follow-up for MR, $44 \%$ of patients demonstrated improvement, $11 \%$ worsening, and $45 \%$ no change. Preoperative ejection fraction, preoperative aortic valve peak gradient, and magnitude of reduction of aortic valve peak gradient were not predictive of improved postoperative MR. In addition, preoperative AVG and change in AVG were not significant predictors of degree of residual MR in our model (Figure 2). Additionally, patient comorbidities, including congestive heart failure, chronic lung disease, renal insufficiency, hypertension, and diabetes did not predict residual MR (Table 2).

\section{Survival}

Overall survival at 5 years postoperatively was $69.1 \%$. Survival of patients who had mild preoperative MR was $71.7 \%$, whereas survival was $61.8 \%$ in patients who had moderate MR, but this difference did not reach statistical significance $(P=.212)$ (Figure 3$)$. In patients whose MR improved postoperatively, 5-year survival was $73.5 \%$, compared with $65.4 \%$ in patients whose MR did not

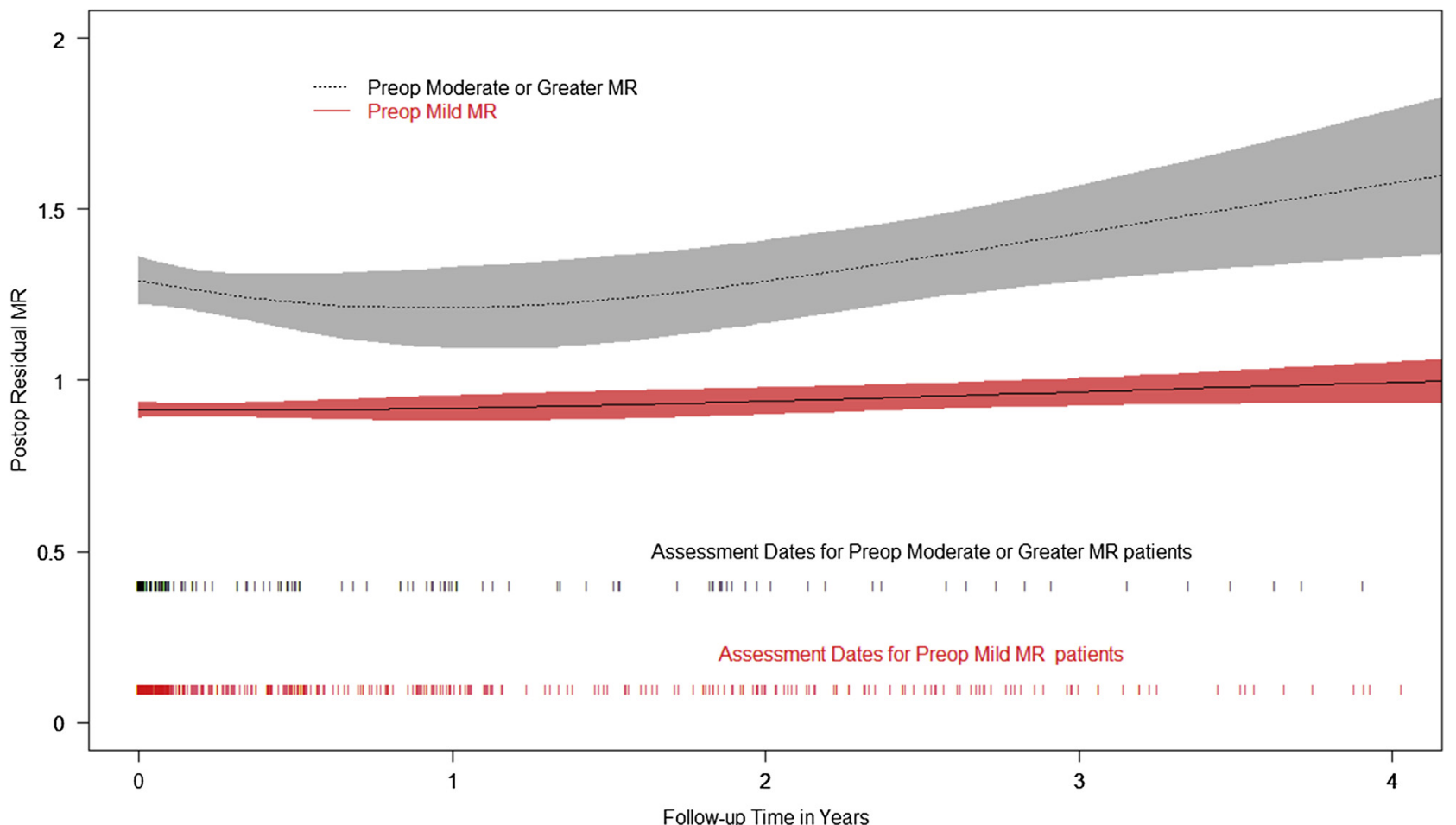

FIGURE 2. Estimated trends in postoperative residual MR for patients with either mild or moderate preoperative MR. Shaded areas bounding the estimated functions for each group show $95 \%$ confidence intervals. The density of echocardiograms over time in each group is represented by the array of short lines corresponding to dates of assessment in each group. Preop, Preoperative; $M R$, mitral regurgitation; postop, postoperative. 
TABLE 2. Patient comorbidities and echocardiographic characteristics and their effects on residual postoperative MR

\begin{tabular}{|c|c|c|c|}
\hline \multirow[b]{2}{*}{ Comorbidity } & \multicolumn{2}{|c|}{ Residual MR at last echocardiogram follow-up } & \multirow[b]{2}{*}{$P$ value } \\
\hline & No improvement $(n=237)$ & Improvement $(\mathbf{n}=186)$ & \\
\hline Reduction in MR (degrees) & $0.18 \pm 0.40$ & $-0.77 \pm .37$ & $<.05$ \\
\hline Residual MR (degrees) & $1.32 \pm 0.56$ & $0.56 \pm 0.40$ & $<.05$ \\
\hline Preoperative LV EF (\%) & $53 \pm 19$ & $50 \pm 13$ & .96 \\
\hline Preoperative peak aortic valve gradient $(\mathrm{mm} \mathrm{Hg})$ & $74 \pm 24$ & $74 \pm 26$ & .98 \\
\hline Reduction in peak aortic valve gradient $(\mathrm{mm} \mathrm{Hg})$ & $-48.8 \pm 24$ & $-51 \pm 26$ & .48 \\
\hline CHF & $103(43)$ & $87(47)$ & .65 \\
\hline Chronic lung disease & $62(26)$ & $52(28)$ & .73 \\
\hline Renal insufficiency & $64(27)$ & $54(29)$ & .75 \\
\hline Hypertension & $169(71)$ & $140(75)$ & .17 \\
\hline Diabetes & $57(24)$ & $47(25)$ & .67 \\
\hline
\end{tabular}

Values are $\mathrm{n}(\%)$, or mean \pm standard deviation, unless otherwise indicated. $M R$, Mitral regurgitation; $L V$, left ventricular; $E F$, ejection fraction; $C H F$, congestive heart failure.

improve $(P=.06)$ (Figure 4). Survival was worse in patients whose MR worsened $(46.7 \% ; P<.01)$.

\section{DISCUSSION}

After AVR for aortic stenosis, the way in which coexistent MR evolves long term is unknown. Prior studies have demonstrated modest, acute improvement in MR after $\mathrm{AVR}$, and more aggressive approaches to MR have been advocated. Yet, no study has specifically examined midor long-term evolution of coexistent MR after AVR. This study found that coexistent moderate MR modestly improves after AVR, but this improvement is not sustained as MR regresses back to baseline within 4 years postoperatively. Moreover, patients with coexistent mild MR at the time of AVR demonstrate minimal improvement in MR.

Preoperative factors, including peak AVG, ejection fraction, and patient comorbidities were not predictive of improvement in postoperative MR. No prior study evaluated the effect of residual MR on postoperative survival. At 5 years postoperatively, no survival difference was found between patients with preoperative mild versus moderate MR; however, patients with improved residual MR

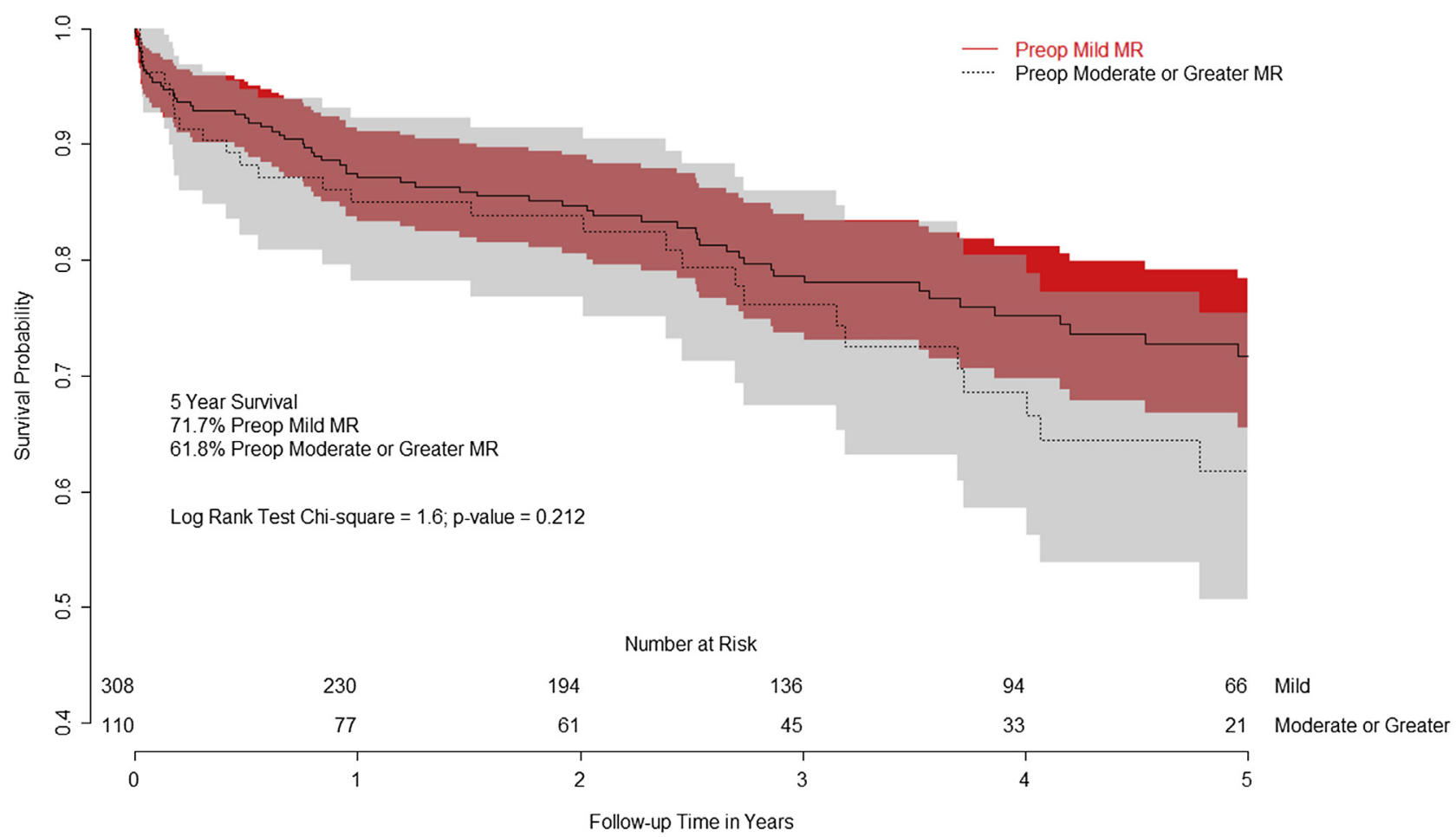

FIGURE 3. Survival distributions are shown, by degree of preoperative MR (mild vs moderate). Survival at 5 years postoperatively was not significantly different between patients with mild versus moderate preoperative MR. Shaded areas bounding the estimated functions for each group show $95 \%$ confidence intervals. Preop, Preoperative; $M R$, mitral regurgitation. 


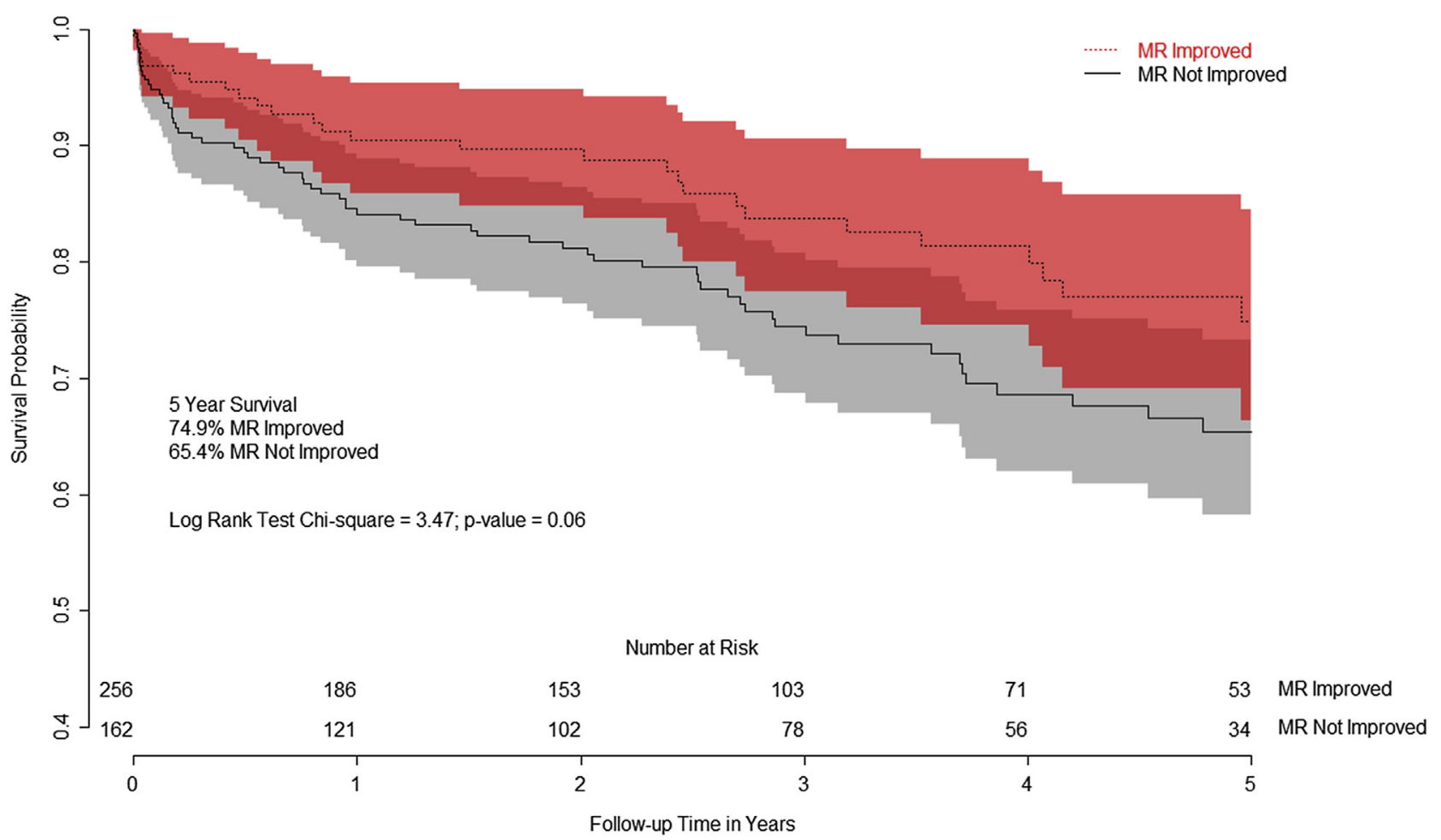

FIGURE 4. Survival analysis of patients with unchanged or improved MR after aortic valve replacement, compared with those whose MR progressed. Survival at 5 years postoperatively was significantly better for patients who had unchanged or improved MR, compared with those whose MR worsened. Long-term survival was significantly better for patients with improved MR, compared with those with worse MR. Shaded areas bounding the estimated functions for each group show $95 \%$ confidence intervals. $M R$, Mitral regurgitation.

demonstrated a trend toward improved survival, compared with patients whose MR either remained unchanged or worsened after AVR.

In patients who had mild MR, very little regression or progression was seen in their MR after AVR. Similarly, Kaczorowski and colleagues ${ }^{4}$ found that patients with mild MR experienced only a one-quarter degree improvement in MR per patient after AVR, and little progression of MR in $<2$ years of follow-up. ${ }^{4}$ These results corroborate the idea that mild MR can be safely observed and not intervened upon at the time of AVR.

Patients with moderate MR have a mean improvement in their MR of 0.56 degrees in the immediate postoperative period. This improvement persists for 2 years after AVR, but it regresses back to preoperative or worse levels in $56 \%$ of patients within 5 years. Other studies that have examined residual MR after AVR in patients who have varying degrees of preoperative MR have demonstrated similar MR improvement, albeit over a much shorter follow-up period.

Moazami and colleagues ${ }^{5}$ found that $45 \%$ of their patients demonstrated marginal improvement in postoperative MR, with a median follow-up time of $818 \pm 752$ days. In fact, $71 \%$ of their patients with severe (4+) MR demonstrated improvement postoperatively, whereas only $43 \%$ of those with moderate (2+) MR had less MR in the postoperative follow-up period. At 1 year after AVR, Eynden and colleagues ${ }^{8}$ demonstrated MR improvement in only onethird of patients who had moderate MR. Similar to our results, the study by Kaczorowski and colleagues ${ }^{4}$ showed that patients with moderate preoperative MR experienced a decrease of MR, of 0.54 degrees per patient, whereas those with mild preoperative MR experienced a decrease of 0.24 degrees per patient.

Although some MR will improve postoperatively, our results demonstrate that this improvement in MR is not durable (Figure 2). By 2 years postoperatively, patients with moderate or greater MR began to regress toward preoperative levels, or in some cases, became worse than they were preoperatively. Even with shorter follow-up periods, other studies were unable to consistently predict durable improvement in MR. ${ }^{3,9}$

To identify the patients who would be more likely to experience progression of postoperative MR, we examined the effects of several preoperative factors and patient comorbidities on postoperative residual MR. Preoperative peak AVG or ejection fraction were not predictive of a reduction in postoperative MR. Additionally, no correlation was found between the reduction in peak AVG and the persistence of MR. This finding suggests that MR may be 
independent of the left ventricular outflow tract gradient; other secondary etiologies, such as left ventricular remodeling from aortic stenosis, may be responsible. Further, patient comorbidities, such as chronic lung disease, congestive heart failure, renal insufficiency, hypertension, and diabetes, did not predict residual postoperative MR in our study.

Other studies have attempted to identify preoperative patient and echocardiographic characteristics that are predictive of postoperative changes in MR. Brasch and colleagues ${ }^{9}$ found that elevated left ventricular mass was the only statistically significant predictor of decreased postoperative MR. The only statistically significant predictor of postoperative MR in multivariate analysis, in a study by Joo and colleagues, ${ }^{10}$ was increased right ventricular systolic pressure. Similarly, Unger and colleagues ${ }^{11}$ published a study indicating that postoperative MR was likely to be improved in patients who had reduced left ventricular function and increased left ventricle size.

Finally, Jeong and colleagues ${ }^{12}$ demonstrated that patients with preoperative atrial fibrillation and an ejection fraction $>40 \%$ were more likely to suffer from residual postoperative MR. Nonetheless, these studies are limited by their short follow-up periods, and the improvement in residual MR in patients with a lower preoperative ejection fraction may be relatively short-lived; it did not persist through the longer follow-up period of our study.

More important, however, than the simple presence of residual MR after AVR, are the structural and hemodynamic consequences of MR, and their effects on longterm survival. Our data demonstrated that no significant difference was found in the 5-year survival of patients, based on their degree of preoperative MR. In this study, at 5 years, patients who had improved postoperative MR demonstrated a trend toward improved survival, compared with those with either no improvement or worsening MR (73.4\% vs $64.5 \% ; P=.06)$. Ruel and colleagues ${ }^{5}$ demonstrated that patients with moderate MR and another risk factor for congestive heart failure, including left atrial diameter $>5 \mathrm{~cm}$, preoperative peak AVG $<60 \mathrm{~mm} \mathrm{Hg}$, or atrial fibrillation, were at increased risk for the development of congestive heart failure, and thus, decreased survival.

Moreover, these effects seem to be magnified in older populations, as moderate MR at the time of AVR has been identified as an independent risk factor for decreased long-term survival. ${ }^{13}$ A corollary to this finding is that decreased residual MR would reduce risk for heart failure and improve survival. The survival trend identified in our patient cohort supports this assumption. Although our study did not identify any preoperative patient characteristics or echocardiographic findings that could reliably predict which patients would experience postoperative progression of MR, the difference in survival favors a more aggressive approach to concomitant mitral valve pathology at the time of AVR.

\section{Limitations}

Our study is inherently limited by its retrospective analysis of data from a single institution. Although echocardiograms were read in a single laboratory, substantial interobserver variability is possible and was not taken into account in the analysis. Additionally, pre- and postoperative MR results were graded, using standard criteria, which assign numeric values to ordinal grades. In this analysis, changes and differences in MR values over time are measured as continuous numeric values. These measures of change are not true continuous variables, but rather simple numeric differences between the ordinal category values established for grading residual MR. Although this scaling practice is inexact, it is widely used for communicating levels of MR change for such patients.

Additionally, we compared the survival of patients with postoperative, improved or stable MR with those who had worse MR over the available follow-up period, with the groups defined according to the last postoperative echocardiogram results. However, differences in the time to improvement are not accounted for in our analysis, because determining exactly when patients improved was not possible. Further analyses using postoperative improvement of MR as a time-varying covariate could provide an improved perspective on the relationship between MR and patient survival.

Another limitation is the possibility of sampling bias toward patients who had a greater degree of residual MR, as each patient did not have regular prescribed echocardiographic follow-up. Further, this study followed survival but not development of heart failure symptoms in patients with residual MR. Finally, this study was not designed to assess the etiology of MR (ie, left ventricular remodeling), which some authors ${ }^{8,14}$ have found to be significant in the persistence of postoperative MR.

\section{CONCLUSIONS}

Coexistent MR improves to a modest extent after AVR, but eventually regresses back to baseline or worsens over time in many patients. Preoperative AVG, reduction of AVG, heart failure, or atrial fibrillation were not predictive of residual MR. Moderate preoperative MR did not adversely affect 5 -year survival. Patients who had improvement in MR, however, demonstrated a trend toward improved survival at 5 years. More aggressive approaches for coexistent moderate MR should be considered in patients who present for AVR for aortic stenosis.

\section{Conflict of Interest Statement}

Authors have nothing to disclose with regard to commercial support. 


\section{References}

1. Harpole DH, Gall SA, Wolfe WG, Rankin JS, Jones RH. Effects of valve replacement on ventricular mechanics in mitral regurgitation and aortic stenosis. Ann Thorac Surg. 1996;62:756-61.

2. Nishimura RA, Otto CM, Bonow RO, Carabello BA, Erwin JP, Guyton RA, et al. 2014 AHA/ACC guideline for the management of patients with valvular heart disease. JACC. 2014;63:75-185.

3. Moazami N, Diodato MD, Moon MR, Lawton JS, Pasque MK, Herren RL, et al. Does functional mitral regurgitation improve with isolated aortic valve replacement? J Card Surg. 2004;19:444-8.

4. Kaczorowski DJ, MacArthur JW, Howard J, Kobrin D, Fairman A, Woo J. Quantitative evaluation of change in coexistent mitral regurgitation after aortic valve replacement. J Thorac Cardiovasc Surg. 2013;145:341-8.

5. Ruel M, Kapila V, Price J, Kulik A, Burwash IG, Mesana TG. Natural history and predictors of outcome in patients with concomitant functional mitral regurgitation at the time of aortic valve replacement. Circulation. 2006;114:I541-6.

6. Zoghbi WA, Enriquez-Sarano M, Foster E, Grayburn PA, Kraft CD, Levine RA, et al. Recommendations for evaluation of the severity of native valvular regurgitation with two-dimensional and Doppler echocardiography. J Am Soc Echocardiogr. 2003;16:777-802

7. Sullivan LM. Statistical primer for cardiovascular research: repeated measures. Circulation. 2008;117:1238-43.

8. Eynden EV, Bouchard D, El-Hamamsy I, Butnaru A, Demers P, Carrier M, et al. Effect of aortic valve replacement for aortic stenosis on severity of mitral regurgitation. Ann Thorac Surg. 2007:83:1279-84.

9. Brasch AV, Khan SS, DeRobertis MA, Kong JHK, Chiu J, Siegel RJ. Change in mitral regurgitation severity after aortic valve replacement for aortic stenosis. Am J Cardiol. 2000;15:1271-4.

10. Joo H, Chang B, Cho S, Youn Y, Yoo K, Lee S. Fate of functional mitral regurgitation and predictors of persistent mitral regurgitation after isolated aortic valve replacement. Ann Thorac Surg. 2011;92:82-8.

11. Unger P, Plein D, Van Camp G, Cosyns B, Pasquet A, Henrard V, et al. Effects of valve replacement for aortic stenosis on mitral regurgitation. Am J Cardiol. 2008; 102:1378-82.

12. Jeong DS, Park PW, Sung K, Kim WS, Yang J, Jun T, et al. Long-term clinical impact of functional mitral regurgitation after aortic valve replacement. Ann Thorac Surg. 2011:92:1339-45.

13. Barreiro CJ, Patel ND, Fitton TP, Williams JA, Bonde PN, Chan V, et al. Aortic valve replacement and concomitant mitral valve regurgitation in the elderly. Circulation. 2005;112:I443-7.

14. Christenson JT, Jordan B, Bloch A, Schmuziger M. Should a regurgitant mitral valve be replaced simultaneously with a stenotic aortic valve? Tex Heart Ins J. 2000;27:350-5.

Key Words: mitral regurgitation, aortic valve, replacement, echocardiography

\section{Discussion}

Dr Ravi K. Ghanta (Charlottesville, Va). Thank you. I would like to thank the association for the opportunity to present our study evaluating the natural history of coexistent MR after AVR. We have no disclosures.

We frequently identify coexistent MR in patients who have aortic stenosis. Many studies show that $50 \%$ to $75 \%$ of patients have some degree of MR at the time of AVR. I think all of us would agree that severe MR necessitates concomitant intervention of the mitral valve at the time of AVR. What to do for lesser degrees of MR, however, is unclear. It is widely assumed that MR improves after AVR. This is entirely logical because MR is at least partly exacerbated by the elevated left ventricular pressure encountered because of the elevated AVG. The AVR would relieve the AVG, and this should, logically, decrease MR.
Few studies, however, have actually evaluated whether this occurs. Dr Woo and colleagues presented at this meeting, 3 years ago, a study evaluating the short-term effects of AVR on MR. They found that MR improved only modestly after AVR. No study, however, has evaluated the long-term effects of AVR on residual MR; thus, the appropriate management of patients with moderate MR remains unclear.

The objectives of this study were to examine the evolution of residual MR following isolated AVR for aortic stenosis. We sought to identify prognostic indicators for improvement in MR after isolated AVR. We also sought to identify the effect of residual MR on survival. We evaluated consecutive patients, from 2004 to 2013, who underwent primary isolated AVR for aortic stenosis, who also had coexistent MR and at least one postoperative echocardiogram performed at the University of Virginia.

Using these criteria, we identified 423 patients. These 423 patients underwent 903 postoperative echocardiograms. The MR was graded using the American Society of Echocardiography scale, which was 0 for no MR, 0.5 for trace MR, 1 for mild, 2 for moderate, 3 for moderate/severe, and 4 for severe. These echocardiograms were obtained at varying time intervals, and the residual MR was modeled utilizing hierarchic generalized linear modeling techniques. We evaluated the relationship of AVG and change in AVG after AVR to residual MR. We divided patients into those that improved postoperatively and those that did not improve postoperatively, in terms of MR, and we compared various preoperative demographic data and hemodynamic data.

Survival data were obtained from our clinical data repository at the University of Virginia, which combines clinical data with Social Security Death Index data and data from the Commonwealth of Virginia, to determine survival. We utilized Cox proportional hazard regression analysis to assess survival in these patients.

Of the 423 patients in this study, 319 had preoperative mild MR, and 105 had preoperative moderate or greater MR. The mean age of patients in the study was 73; the mean AVG was 47.9. The majority of patients in this study had functional MR-96\%. A few had rheumatic or leaflet prolapse. Comparison of the moderate to the mild MR patients preoperatively showed that the moderate patients were more likely to be female ( $53 \%$ vs $36 \%$ ), more likely to have atrial fibrillation ( $40 \%$ vs $29 \%$ ), more likely to have a preoperative diagnosis of heart failure $(70 \%$ vs $50 \%$ ), and more likely to have a higher preoperative pulmonary artery pressure (51 vs $40 \mathrm{~mm} \mathrm{Hg}$ ).

Figure 2 shows, on the y-axis, the median change in MR from baseline versus time, and 2 groups are shown-those that had preoperative mild MR, and those that had preoperative moderate or greater MR. The acute reduction in MR in patients who had preoperative mild MR was 
0.13 degrees-so, a very small change. The acute reduction in MR in patients who had moderate MR was approximately 0.53 degrees.

At 1 year postoperatively, MR begins to worsen, and by year 4, the change in MR from baseline approaches 0 , indicating that many patients return to the baseline level of MR they had prior to AVR. This figure shows the same data in a different way. The y-axis is the absolute value of the MR grade versus time, for the 2 groups - the moderate or greater MR, and the mild MR, and here you see the mild MR patients stay close to mild, and the moderate or greater MR patients initially come to a level of 1.3 and begin to worsen over time. An important finding is that $56 \%$ of patients at the conclusion of the study had the same or worse MR as they did preoperatively.

We stratified the patients, by no improvement in their MR versus improvement in their MR; 186 patients had improved MR, and 237 had no improvement or worsening of MR. The improved patients had a median improvement of 0.77 degrees. The nonimproved patients had a median increase in MR of 0.18 degrees, and residual MR was 0.56 in the patients that had improvement. There were no statistically significant differences between these 2 groups in left ventricular ejection fraction, preoperative AVG, change in AVG after AVR, or these other comorbidities.

We also performed a linearized model to look at the relationship of change in AVG to reduction of MR and found no statistically significant relationship. Figure 3 shows survival as a function of time for the 2 groupspatients who had preoperative mild MR and those that had preoperative moderate or greater MR. The 5-year survival was $71.7 \%$ versus $61.8 \%$. This was not statistically significant. So the grade of preoperative MR did not influence 5-year survival in this study. We looked at the survival in patients that had improved MR, versus those that did not have improved MR; the 5-year survival was $74.9 \%$ versus $65.4 \%$. This difference did not achieve statistical significance, but the $P$ value was very close, at .06 .

This is a limited study. It is a single-center study, retrospective. By the nature of that type of study, the echocardiography follow-up was irregular, and it was dependent on the practices of the cardiologists. We, of course, would expect some sampling bias, as sicker patients probably were more likely to get an echocardiogram than those who were doing well, and we did not assess the symptoms of residual MR in this study; we also did not evaluate the electrocardiograms for other factors, such as ventricular remodeling.

But from these data, we conclude that coexistent MR only modestly improves after isolated AVR for aortic stenosis, and in many patients, it eventually regresses back to baseline or worsens. Preoperative AVG, reduction of valve gradient, heart failure, and atrial fibrillation were not predictive of degree of residual MR; the degree of preoperative MR in this group of patients did not adversely affect 5-year survival. Patients with improvement in MR after AVR did demonstrate a trend toward improved survival at 5 years, and my assumption would be that if this study was further followed, the differences between the 2 groups would widen, and a statistically significant result would likely be obtained. So we would ultimately conclude that more aggressive mitral valve surgical options should be considered in select patients who have moderate or greater MR and are in need of AVR for aortic stenosis. Thank you very much. This discussion will be led by Joseph Woo.

Dr Joseph Woo (Palo Alto, Calif). Thank you. So, Dr Ghanta, I congratulate you on your outstanding clinical research effort, and it is particularly reassuring to see that your MR reduction findings nearly identically mirror those reported by other groups. But your study goes further, and I commend your tracking of the longitudinal outcomes with respect to MR recurrence, as well as survival, and it is particularly notable to see that trend in difference in survival depending upon what happens to your MR. With your permission, I would like to incorporate your data on Monday morning, when we present to the National Institutes of Health Cardiothoracic Surgery Network in Bethesda a proposal to fund a multicenter, prospective, randomized trial on this topic. So I have 3 specific questions.

The first relates to anatomic criteria. So, have you looked at your echocardiograms and identified any potential structural features of the mitral valve, such as leaflet or annular calcification, that may predict a greater likelihood of not having reduction in MR after AVR?

Dr Ghanta. Excellent questions, and yes, I would be more than happy to share these data, and I do think a prospective study would be exactly what is required to evaluate this question. Although these retrospective studies have numerous limitations, primarily in sampling bias, with echocardiograms, there is a wealth of information that I think can be gained. There obviously are factors that should predict who will improve, versus who will not improve. We just have not identified them yet, and it is, I think, factors that you pointed out in terms of evaluating the mitral annulus size and dimensions, left ventricular size and dimensions, and left atrial size and dimensions-all would likely be predictive. We have not looked at the echocardiograms in for that type of analysis. It is fairly resource intensive to do that, and we would love to do that in the future.

Dr Woo. So, one of the concerns is that escalating the operation somewhat by adding a mitral intervention will increase the perioperative morbidity and mortality, and may not yield benefit long term. You have shown the impact of not doing something, but have you looked at any of your 
groups of patients when they underwent an AVR as well as a mitral valve repair or replacement for MR, not primary stenosis, mitral stenosis, but for MR and see if they had any differences in outcomes compared to your AVR-only group?

Dr Ghanta. We have not made that comparison. I think we, like probably most surgeons, would have a bias to just do an AVR in someone who has moderate MR, and so it is hard to compare the two, because they are different degrees of disease. The reason is that we would never not do a mitral valve operation if someone had aortic stenosis and severe MR, but it would be something that we could obtain from a trial such as you are suggesting.

Dr Woo. Great, and given the significance of your findings, can you give us an idea of whether this has influenced the clinical practice at the University of Virginia?

Dr Ghanta. Not yet. I think my own personal practice would be that if I had a young patient who had aortic stenosis and had at least moderate, but probably more moderate/severe MR, I would take a closer look at the electrocardiogram to see what I could do to reduce the MR for the long term in this patient, probably a ring annuloplasty, but at this point, we have not changed our practice based on these data.

Dr Woo. Thank you again for sharing that excellent body of work.

Dr Richard Shemin (Los Angeles, Calif). I would like to understand your cohort in a broader context. So, how many patients at the University of Virginia during the same time period got an isolated AVR, but actually got a procedure on the mitral valve, particularly if they had moderate or greater degree of MR?

Dr Ghanta. I do not have that number. If someone has aortic stenosis and moderate MR, it would be unlikely that they had a mitral valve operation, unless they had a structural mitral valve defect, such as a prolapse or a ruptured cord.

Dr Shemin. Okay, so that was an institutional philosophy and approach. What about severe MR?

Dr Ghanta. The numbers, I do not know, but the institutional approach would be to fix the valve either with a repair, ideally, or replacement if necessary.

Dr Shemin. Okay, so in other words, this was consistent across the practice of all surgeons at the University of Virginia-to leave moderate degrees of MR if it was thought to be functional in the setting of critical aortic stenosis.

Dr Ghanta. Correct.

Dr Shemin. Thank you.

Dr Ghanta. I think I would just add that we do this because there is the assumption that the MR will get better; I think what we have learned from these data, as we have from other studies, is that it does not.
Dr Shemin. Ravi, congratulations, that was very well presented. I think in pediatric heart surgery, we sort of learned the right-sided version of this - that when we were reoperating for tetralogy, if somebody had mitral, sort of moderate plus tricuspid regurgitation, the assumption, maybe 15 or 20 years ago, would have been: "Well, when you reduce that volume load, you put in a valve that is going to get better," and we know now that it does not. So that sort of played into what happens now with Melody (Melody valve; Medtronic, Minneapolis, Minn) implantationright? Because a pediatric cardiologist is more likely to refer a patient for surgical repair if the patient has moderate-tosevere tricuspid regurgitation, for a concomitant 2-valve procedure or repair, and then pulmonary valve repair, versus just putting in a valve or Melody, or whatever you decide to do. So I am wondering-I know that you have a transcatheter aortic valve replacement program. I was wondering how this is connected to that — because the same philosophy would dictate that as we lower our-not our expectationsbut our threshold for aortic transthoracic aortic valve replacement, what you do with the mitral valves is going to become incredibly important, as far as exposing the patient to surgery.

Dr Ghanta. That is an excellent question. Listening to other talks, it seems that a lot of native valve aortic stenosis will be treated with TAVR in the future. The basic concept, though, of this study, is that the MR will continue and will need further follow-up and evaluation, possibly intervention. It could be a TAVR, plus a MitraClip (Abbott Vascular, Abbott Park, Ill), for example. I am not advocating that that be the approach, but there would be some type of intervention needed for the mitral valve, regardless of how the aortic stenosis is alleviated.

Dr Craig Selzman (Salt Lake City, Utah). I am a little confused looking at one of the tables and trying to figure out what I am going to take home from this, because it looks like you combined the mild and the moderates with the improvements? Maybe I read that incorrectly. So, when you said "improvement versus nonimprovement" - for those that improved, you included both mild and moderate. So are there certain factors about thosewhen you looked at just the folks that were preoperative moderate that improved - that you could identify? Does that make sense?

Dr Ghanta. Yes. I understand what you are saying, and you are right. That table that showed improved versus not improved included both preoperative mild and moderate, and I think we did not analyze it to look at just the moderate preoperative patients who improved, versus the nonimproved, but that is an excellent idea, and something we will pursue.

Dr Patrick Griffith (Marysville, Calif). Excellent paper. We all struggle with this phenomenon. My concern is that it was very subjective, in terms of saying mild and moderate. I 
always try to say: Do we have something else that is more objective, with things such as regurgitant orifice area or vena contracta that were measured, and from that, what do we do moving forward? Is there a regurgitant orifice area or vena contracta or other objective measurement that you use to say: "Yes, this is moderate, and this is our cutoff point"?

Dr Ghanta. You bring up an excellent point. We are using the more qualitative assessment of MR, as opposed to the quantitative assessments using effective regurgitant orifice area, and I definitely think that in a prospective study, that should be how it is evaluated. For many reasons, we use this measure because it is the most commonly available and the most commonly utilized. There is going to be some intercardiologist variability in terms of reading these studies, but fortunately, we have a group of cardiologists at the University of Virginia that are focused on echocardiography, and this handful of cardiologists have read all the reports. So there would be some consistency from reading to reading, but you do raise a good point, and a more quantitative measure would be more ideal.

Readers who found these articles interesting may also like to read the following papers found in recent and future issues of our sister publications, Seminars in Thoracic and Cardiovascular Surgery and Operative Techniques in Thoracic and Cardiovascular Surgery!

\section{Acquired: Aortic Disease}

Original Submission: Outcomes of Open Surgical Repair for Type B Dissecting Aortic Aneurysm with Alternative Methods in the Endovascular Stent Era. Mitsumasa Hata. Semin Thorac Cardiovasc Surg 2015; Summer; 27(2):106-112.

Editorial Commentary: It is Difficult to Compare Apples and Oranges: Acute and Chronic Type B Aortic Dissections, Complicated and Uncomplicated, are Different and Should be Treated as Such. Ourania Preventza. Semin Thorac Cardiovasc Surg 2015; Summer; 27(2):113-114.

News and Views: Treatment of Thoracic Aortic Aneurysm: Role of Earlier Intervention. Bulat A. Ziganshin. Semin Thorac Cardiovasc Surg 2015; Summer; 27(2);135-143. 


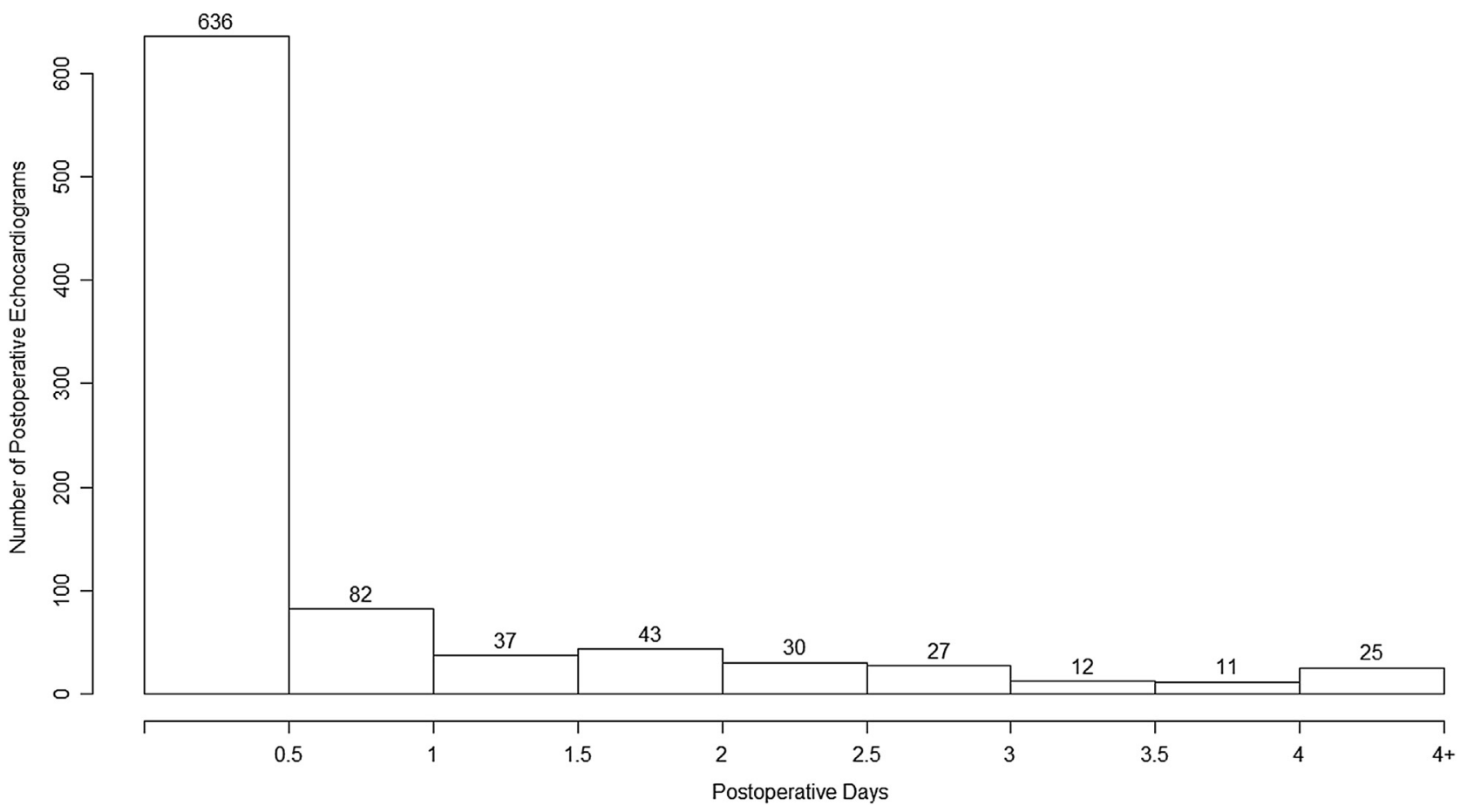

FIGURE E1. Summary of echocardiogram follow-up frequencies and durations. 